ISAHP Article: Jantscher, Schwarz, Zinser/Development of an Innovative AHP-Based Decision Support System in the Field of IT Service Management/To Be Submitted to the International Symposium of the Analytic Hierarchy Process 2014, Washington D.C., U.S.A.

\title{
DEVELOPMENT OF AN INNOVATIVE AHP-BASED DECISION SUPPORT SYSTEM IN THE FIELD OF IT SERVICE MANAGEMENT
}

\author{
Martin Jantscher* \\ FH JOANNEUM University of Applied Sciences \\ Graz, Austria \\ E-mail: martin.jantscher@edu.fh-joanneum.at \\ Christopher Schwarz \\ FH JOANNEUM University of Applied Sciences \\ Graz, Austria \\ E-mail: christopher.schwarz@fh-joanneum.at \\ Erwin Zinser \\ FH JOANNEUM University of Applied Sciences \\ Graz, Austria \\ E-mail: erwin.zinser@fh-joanneum.at
}

\begin{abstract}
The order in which service engineers in the field of IT service management decide to resolve incidents is crucial concerning the impact on the business performance of the IT service provider. A possible solution to reduce negative impacts is to support service engineers by means of a decision support system that calculates priorities for the incidents based on their business impact. Considering these priorities in their decisions, service engineers can help reduce such negative impacts on the IT service provider's business.

The aim of this study is to incorporate the Analytical Hierarchy Process (AHP) method into a software to prioritize incidents according to the severity of their business impact.

As a result a decision support system called Incident Prioritizer (IP) was developed. It uses the AHP method to calculate priorities for incidents based on typical criteria that are relevant in the field of IT service management to assess the business impact of incidents. These criteria are commonly defined by the management of an IT service provider organization and reflect the management's understanding of the business impact that is caused by incidents.

The results of the work suggest that the AHP method can be successfully applied for the given problem of incident prioritization. A decision model was introduced that consists of one level of criteria that should reflect the factors that contribute to the business impact of incidents. The introduced decision model was intentionally kept simple by using only a few relevant criteria. At the same time it is extensible by adding further criteria as needed.
\end{abstract}

Keywords: Analytic Hierarchy Process, IT Service Management, Decision Support System, Incident Prioritizer, ITIL. 
ISAHP Article: Jantscher, Schwarz, Zinser/Development of an Innovative AHP-Based Decision Support System in the Field of IT Service Management/To Be Submitted to the International Symposium of the Analytic Hierarchy Process 2014, Washington D.C., U.S.A.

\section{Introduction}

In the era of Cloud Computing the management of information technology (IT) infrastructure becomes an ever increasing challenge. Especially large IT service providers have to find ways to manage complex, centralized and large-scale IT infrastructure. The service providers get support in their endeavor by consulting best practice guidelines on managing IT infrastructure effectively such as IT infrastructure library (ITIL).

It is a common practice for IT service providers to issue contracts to customers that specify the IT service offered as well as certain service level targets that the IT service provider has to meet. Furthermore, the contract specifies penalties that are due in case the IT service provider fails to meet those service level targets.

It is also common for IT service providers to employ service engineers that are responsible for resolving incidents. Incidents are basically interruptions of IT services. These interruptions can have various causes such as hardware failure or software crashes. Large IT service providers face dozens of incidents each day that often occur at the same time.

Typically, service engineers decide in which order they resolve those incidents. Without any information on the penalties that are caused by the incidents the order in which service engineers decide to resolve incidents can have a severe negative impact on the business of IT service providers (Beims, 2010).

A possible solution to close this information gap is to provide the service engineers with a decision support system that prioritizes incidents based on the severity of their business impact.

The overall goal of this study is to incorporate the Analytical Hierarchy Process (AHP) method into an ITIL-compliant software to prioritize incidents according to the severity of their business impact. The AHP is a widely accepted approach for decision making in different domains. Consequently, it is also an adequate approach for the purposeful prioritization of incidents.

The goal of this study was achieved by developing a software called Incident Prioritizer (IP). The IP uses the AHP to calculate priorities for incidents based on well-defined decision criteria. It is a decision support system that supports the aforementioned decision process of service engineers by prioritizing all open incidents according to their business impact. To assess the business impact of incidents multiple criteria are taken into account. These criteria are commonly defined by the management of an IT service provider. The output of the IP is prioritized incidents. The calculated priorities represent the business impact of the incidents. Considering these priorities in the decision process of the service engineers helps reducing negative impact on the business of the IT service provider.

\section{Literature Review}

It is a well-known fact in the field of IT service management that incidents exhibit a negative impact on the business performance of IT service providers. The ITIL guideline thus recommends IT service providers to prioritize their incidents based on their business impact (OGC, 2007). Business impact includes factors like financial losses or the number of customers that are affected by an incident. In addition to that (Beims, 2010) states that the order in which service engineers decide to resolve incidents can also contribute to a negative impact on the business of the IT service provider. 
ISAHP Article: Jantscher, Schwarz, Zinser/Development of an Innovative AHP-Based Decision Support System in the Field of IT Service Management/To Be Submitted to the International Symposium of the Analytic Hierarchy Process 2014, Washington D.C., U.S.A.

There is a lot of ITIL-compliant software commercially available that is used by IT service providers to support their business (ITIL, 2014). However, to our best knowledge no ITIL-compliant software exists that relies on a mathematical foundation for prioritizing incidents.

\section{Objectives}

The objective of this study is to incorporate the AHP method into an ITIL-compliant software to prioritize incidents according to the severity of their business impact. The objective was achieved by developing a decision support system called Incident Prioritizer. The following sections will elaborate on the Incident Prioritizer software and its AHP-based prioritization capabilities.

\section{Research Design/Methodology}

Figure 1 shows a highly simplified version of the software architecture that the Incident Prioritizer is part of.

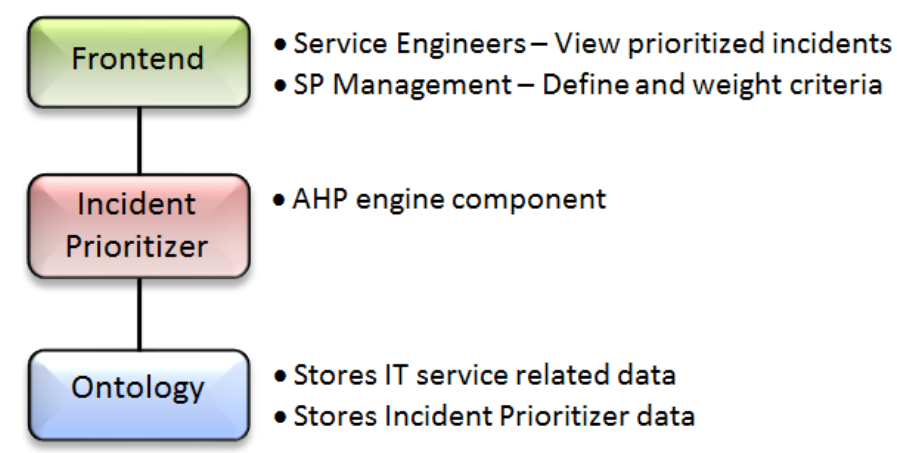

Figure 1 Simplified software architecture of the Incident Prioritizer

Figure 1 shows a software architecture that consists of three modules (Frontend, Incident Prioritizer, Ontology) that are connected with each other. The IP module is the one developed for this study. The other two modules are published elsewhere but are required for the proper functioning of the IP.

The Ontology can be seen as a central database that stores IT service related data such as incidents, customers, contracts with their service level targets and penalties. Furthermore, it stores the criteria and weighting data that are considered by the IP in its AHP-based calculation of priorities.

The IP module uses the Ontology module as its data source. The IP module incorporates an AHP engine that is used to calculate priorities based on the data that comes from the Ontology.

The IP module is controlled via the Frontend module. The Frontend module provides a graphical user interface for using the functionality of the IP module. It is used by both service engineers and the management of IT service providers. The service engineers can trigger the prioritization process on the IP and get to see the results of this process which is prioritized incidents. The management of the provider can choose the criteria that are used to assess the severity of the business impact of incidents. They can also carry out a pairwise comparison process there to obtain the weighting of the criteria based on Saaty's scale of absolute numbers (Saaty, 2008).

$\begin{aligned} & \text { International Symposium of } \\ & \text { the Analytic Hierarchy }\end{aligned}$
$\begin{aligned} & \text { Process } \\ & \text { Pro } 29-\text { July 2, 2014 }\end{aligned}$


ISAHP Article: Jantscher, Schwarz, Zinser/Development of an Innovative AHP-Based Decision Support System in the Field of IT Service Management/To Be Submitted to the International Symposium of the Analytic Hierarchy Process 2014, Washington D.C., U.S.A.

The IP calculates priorities on the basis of an AHP decision model. This model has the goal of finding the incident with the greatest business impact. Thus the decision model considers incidents as its alternatives.

It is important to understand that the decision model used in the IP is not static. It varies depending on the management's preferences regarding criteria as well as on the current open incidents of an IT service provider organization.

The criteria that can be used in the decision model have been derived from expert knowledge. The expert is working at a large IT service provider organization in Austria that serves customers all over the globe. Based on interviews with the aforementioned expert the following four criteria have been developed:

- NoOfCustAffected - The number of customers affected by an incident.

- IsImpCustAffected - Checks whether customers marked as important are affected by an incident.

- Penalty - The total penalty caused by an incident.

- RemainingTime - The time remaining until a service level target is violated.

The judgments of the criteria with respect to the goal have to be carried out on an individual basis by the management of the IT service provider that is using this decision support system. This is done by using the Frontend module shown in Figure 1. The judgments made there shall reflect the management's understanding of what contributes most to the business impact caused by incidents.

The judgments of the alternatives with respect to the criteria are carried out automatically by the IP based on data that is related to the incidents. This data is obtained from the Ontology module shown in Figure 1.

\section{Data/Model Analysis}

As already mentioned in the previous section the decision model that the IP software uses to calculate priorities varies in terms of its criteria, their weightings and the alternatives. Figure 2 shows a representative example of an AHP decision model that can be successfully processed by the IP software to calculate priorities.

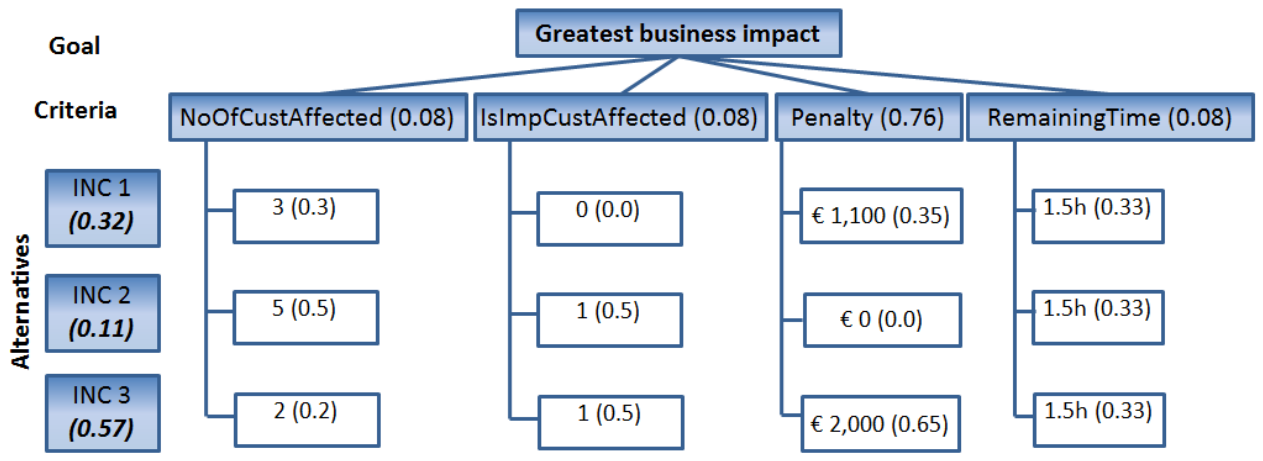

Figure 2 Representative example of an Incident Prioritizer compliant AHP decision model

Figure 2 shows that the service provider management decided to consider all of the four well-defined criteria in the prioritization process. The criteria weightings that the management made in this example are shown in brackets next to the name of the criteria (e.g. 0.76 is the priority for criterion "Penalty"). It seems that the management puts the 
ISAHP Article: Jantscher, Schwarz, Zinser/Development of an Innovative AHP-Based Decision Support System in the Field of IT Service Management/To Be Submitted to the International Symposium of the Analytic Hierarchy Process 2014, Washington D.C., U.S.A.

highest priority on the criterion "Penalty" when it comes to assess the business impact of incidents. Figure 2 shows three incidents as alternatives that have to be prioritized. The white boxes that are connected to the upper level criteria represent data of the incidents (e.g. 3, 5, 2 for the criterion "NoOfCustAffected"). The content of the top left white box in Figure 2 has the following meaning: Incident 1 affects 3 customers. Compared to the other two incidents and how many customers they affect the priority of incident 1 with respect to the criterion "NoOfCustAffected" is 0.3 . The priorities of incidents are also shown in brackets underneath the name of the incidents (e.g. 0.57 is the final priority for incident "INC 3"). All calculation of priorities is done by the AHP engine component of the IP module.

As already mentioned in section 4 of this paper the values used for comparing alternatives with respect to criteria do not come from human beings judgments. Instead the judgments are based on the data of the Ontology component shown in Figure 1. The judgments are obtained by putting the data of incidents in relation to each other. In Figure 2, lets compare INC1 to INC2 with respect to the criterion "NoOfCustAffected". The data for incident 1 with respect to "NoOfCustAffected" is 3 . The data for incident 2 is 5 . The judgment is obtained by dividing the data of incident 1 by data of incident $2(3 / 5)$. This step is carried out by the IP module.

\section{Limitations}

So far the developed decision support system was tested with only three synthetic incidents. However, in a real business scenario the system has to handle dozens or even hundreds of incidents which might lead to a non-deterministic behavior of the software module.

Another limitation refers to the consistency of judgments made within the IP software. By design of the IP judgments are made on the criterion level by human beings as well as by the software itself on the alternatives level. So far the IP does not check the consistency of the judgments on any of those two levels.

\section{Conclusions}

In this study a decision support system called IP was developed. It implements the AHP method for the purpose of prioritization. The IP is an ITIL-compliant software that is used by IT service providers to prioritize incidents based on their business impact.

Future research has to test the developed decision support system in a real business scenario to find out whether the software provides meaningful results when facing the challenge of prioritizing dozens of incidents. Another suggestion for further research is to check for the consistency of judgments that are considered in the prioritization process.

\section{Key References}

Saaty, T.L. (2008). Decision making with the analytic hierarchy process, Int. J. Services Sciences, Vol. 1, No. 1, pp.83-98.

Beims, Martin. (2010). IT-Service Management in der Praxis mit ITIL 3. Hanser Verlag, Munich.

OGC. (2007). ITILv3 - Service Operation. The Stationary Office, Norwich.

ITIL. (2014). Endorsed Software Tools. Retrieved March 31, 2014, from http://www.itilofficialsite.com/SoftwareScheme/EndorsedSoftwareTools/EndorsedSoftwareTools.aspx 